\title{
Tackling water pollution: real-time monitoring of residual antimicrobials concentration in aquaculture with microwave spectroscopy
}

\author{
Olga Korostynska ${ }^{1 *}$, Worawat Jansomboon ${ }^{2}$, Ismini Nakouti ${ }^{3}$, Alex Mason ${ }^{4}$ and Ahmed Al- Shamma'a \\ ${ }^{1}$ Faculty of Engineering and Technology, BEST Research Institute, Liverpool John Moores University, Liverpool, UK \\ ${ }^{2}$ Department of Chemical Engineering, Faculty of Engineering, Kasetsart University, Thailand \\ ${ }^{3}$ School of Pharmacy and Biomolecular Sciences, Faculty of Science, Liverpool John Moores University, Liverpool, UK \\ ${ }^{4}$ Animalia AS, Norwegian Meat Research Institute, 0513 Oslo, Norway
}

\begin{abstract}
To prevent the improper use of antimicrobials in aquaculture and to assist the food safety law enforcement, this paper reports on a bespoke electromagnetic (EM) wave sensing method for real-time in situ monitoring of residual antimicrobials concentrations in water samples. The antimicrobials solutions were tested in bespoke microwave cavity. Transmitted and reflected power signals were analysed in $\mathrm{GHz}$ frequency range and these were dependent on both: the type of antimicrobials present in water and on their concentration.
\end{abstract}

\section{Introduction}

Antimicrobial Resistance (AMR) is usually described as the ability of microorganisms to resist antimicrobial treatments, especially antibiotics. AMR manifests itself as the failure in the treatment of infectious diseases in human and animal health, which also carries a heavy economic cost.

Antimicrobial therapy can be one of the most effective management responses to emergencies associated with infectious disease epizootics, if the antibiotics are correctly chosen and dose is appropriate for the circumstances. Wrong choice or dose both can lead to catastrophic consequences in a long run. The over-use of antimicrobial agents has the potential to increase the frequencies of bacterial resistance and this would have a negative impact on the subsequent use of these agents to control infectious disease in aquaculture [1].

Once the antimicrobials are present in fish, meat or any food products - these can have direct adverse impact on human health. Screening of food products from animal origin for the presence of antimicrobial residues started soon after the introduction of antibacterial therapy in veterinary medicine. From the early 1970s regulatory residue screening in slaughter animals and later in fish became more commonly introduced across the European Union.

In the aquaculture industry around the world antibiotics are used to treat bacterial infections in fish and as prophylactics [2]. This is due to the fact that farmed fish have lowered host defences as a consequence of culturing at high density in a confined space. Norway, United States and Canada allow the use of oxytetracycline in aquaculture and Norway also permits the use of quinolones for aquaculture purposes. Chile one of the largest producers of farmed salmon permits the use of quinolones, oxytetracycline, erythromycin and amoxicillin amongst many other antimicrobial compounds [3]. Antimicrobials are normally administered to all fish (not just the diseased) in food and any unconsumed medicated pellets will naturally settle down at the bottom of the farming site. Also unmetabolised and metabolised antimicrobials will be secreted by the fish in the form of urine and faeces. High residual levels of those antimicrobials may contaminate natural water resources as well as soil, aquatic animals and plants.

Antimicrobials will remain in the sediments for a long period and this process will apply selective pressure for resistant bacteria, posing a dire threat to global health $[4,5]$. For example, large use of quinolones has been linked with an increase of antimicrobial resistance in foodborne pathogens which can be passed on to humans. Exposure of excess levels of antibiotic residues in food can cause allergic reactions in some hypersensitive persons and may affect the human immune system. Similarly, drug and multi-drug resistance in pathogens in human body can also be a serious problem due to the low-dose poisoning in foodstuffs for a long period [6].

There are several methods of quinolones determination in environmental waters, including wastewaters, ground, natural and surface waters, but most of them are lab-based. They require the extraction processes followed by determination and confirmation methods such as variations of liquid chromatography in tandem with fluorescence-mass spectrometry (MS) [7]. The key challenge is

${ }^{\star}$ Correspondence to: Olga Korostynska, Faculty of Engineering and Technology, BEST Research Institute, Liverpool John Moores University, Liverpool, UK, Tel: +44-151-231-2807; E-mail: o.korostynska@ljmu.ac.uk

Key words: antimicrobial resistance; electromagnetic waves; microwave cavity; antibiotics detection; aquaculture monitoring; real-time sensing

Received: April 04, 2018; Accepted: April 20, 2018; Published: April 23, 2018 
implementing a system that can effectively determine quinolones in real time.

This paper reports on the feasibility of using real-time electromagnetic wave sensing at microwave frequencies with nonthermal energy. The approach was previously successfully tested on wastewater chemicals detection [8], in particular $\mathrm{NO}_{3}$ and chemical oxygen demand [9]; for vegetable oil type verification to comply with food labelling regulations [10], in food quality monitoring [11] and in the biomedical area $[12,13]$. This paper communicates the experimental results of using a bespoke microwave cavity for determination of quinolones, in particular enrofloxacin (ENR) and norfloxacin (NOR) antibiotic concentrations. Quinolones are a group of broad spectrum antibiotics that are prescribed for the treatment of drug resistant hospital acquired bacterial infections.

\section{Antimicrobial Resistance in Aquaculture}

Substantial increase of bacteria resistant to quinolones, amoxicillin and oxytetracycline in fish farms employing such antimicrobials has repeatedly been discovered. This clearly demonstrates the Quinolone Resistant (PMQR) genes have been identified in a number of aquatic bacteria [14].

Moreover, scientists strongly believe that residual antimicrobials in the sediments have enough biological activity to stimulate bacterial mutagenesis and horizontal gene transfer (HGT) [15]. Conditions in aquaculture favouring HGT also include the bacterial biofilm formation on the sediments and the antimicrobial resistance associated with the presence of bacteriophages $[4,16]$. The accumulation of antimicrobial resistant genetic elements in aquaculture attracts significant interest as this environment also favours multiple antimicrobial resistance to compounds not even present in the area [17]. These resistant organisms may stay in the sediment or leach out to the environment and pose risk for public health.

\section{Modern Methods of Antimicrobials Detection in Aquaculture Industry}

Most methods currently used for antibiotics detection require the extraction processes followed by determination and confirmation methods such as variations of liquid chromatography (including Ultra Performance Liquid Chromatography (UPLC)) combined with mass spectrometry [7]. Microbial inhibitions assays methods are still widely used for the detection of antibiotic residues. . The tube test and the multi-plate test are the two main test formats, which have the potential to cover the entire antibiotic spectrum within one test, while remaining cost-effective. A tube (or vial, or ampoule) test consists of a growth medium inoculated with spores of a sensitive test bacterium, supplemented with a $\mathrm{pH}$ or redox indicator. At the appropriate temperature, the bacteria start to grow and produce acid, causing an associated change in colour. The presence of antimicrobial residues will prevent or delay bacterial growth, and thus is indicated by the absence or delay of the colour change.

For example, the determination of erythromycin in medicated salmonid fish feed was done using liquid chromatography and UV spectroscopy (LC-UV method) [18]. This method produced high accuracy, $82-90 \%$, for both salmon and trout feed that represented varied pellet sizes and ingredient amounts. The intraday and interday precisions, at $\leq 6$ and $5 \%$, respectively, indicated the method's good repeatability. However, the method is time-consuming as it requires erythromycin to be extracted from feed with acetonitrile and water; then cleaned up by SPE; evaporated to dryness, reconstituted, and only afterwards analysed by LC-UV [18]. Needless to say, an expert is required to perform this analysis and this approach cannot be considered as ready-to-use alternative for instant fish products quality monitoring.

Fast screening immunoassay of sulfonamides in commercial fish samples was reported in [19]. In this approach an indirect competitive enzyme-linked immunosorbent assay (ELISA) was developed in plate to detect three sulfonamide residues (sulfamerazine (SMR), sulfadimetoxine (SDM), and sulfadiazine (SDZ)) in gilthead sea bream (Sparus aurata) samples using different extraction methodologies. The assay detection limits for these antibiotics were lower than $100 \mathrm{\mu g} \mathrm{kg}^{-1}$ (maximum residue level established by the European Union). Notably, this approach is yet to see wide-scale commercial implementation, not least due to concerns with matrix effects for quantification purposes [19].

There are some attempts to develop an in-situ sensor system for quality monitoring of aqueous media and fish products. For example, a stainless steel veterinary syringe coated with a selective membrane of PVC serving was used as a potentiometric detector in a flowinjection-analysis system in an attempt to develop a rapid automated method for on-site determination of sulfadiazine in fish farming [20]. Antibiotic Sulfadiazine is used as a veterinary drug in fish farming, and its monitoring in the tanks is fundamental to controlling the applied doses and avoiding environmental contamination. Reportedly, the best performance of this system was obtained for sensors of $1.5 \mathrm{~cm}$ length and a membrane composition of 33\% PVC, 66\% o-nitrophenyloctyl ether, $1 \%$ ion exchanger, and a small amount of a cationic additive [20]. It exhibited Nernstian slopes of $61.0 \mathrm{mV}$ decade- 1 down to $1.0 \times 10^{-5}$ mol L-1, with a limit of detection of $3.1 \times 10^{-6} \mathrm{~mol} \mathrm{~L}^{-1}$ in flowing media.

Notably, detection of antibiotics using carbon nanotube (CNT)based sensors was attempted in [21]. CNT-based sensor transducers were functionalized with the single-chain variable-fragment $(\mathrm{scFv})$ of antibodies that can selectively bind to a specific antibiotic or the certain family of antibiotics. These CNT-based sensors were functionalised with $\mathrm{A} 2 \mathrm{scFv}$ and $\mathrm{F} 9 \mathrm{scFv}$ and exhibited the specific detection of enrofloxacin or the family-selective detection of fluoroquinolone-based antibiotics, respectively, in a real-time manner.

In another alternative approach to detect antibiotics, a high-density optical microarrays based on molecularly imprinted microsphere sensors that directly incorporate specific recognition capabilities was used to detect enrofloxacin [22]. This work focuses on the detection of Enrofloxacin and Norfloxacin. Enrofloxacin is an antibiotic widely used for both human and veterinary applications. Novel cost-effective approach is reported, in which non-ionising, athermal electromagnetic waves are used as a sensing and transducing mechanism of monitoring the concentration of antibiotics in aqueous solution in real-time.

\section{Electromagnetic Wave Sensing}

Electromagnetic wave sensing at microwave frequencies is a new technological approach which has been successfully used for monitoring solution concentrations [23], fluid level measurements [24], material moisture content $[25,26]$, for continuous process monitoring of biogas plants [27], for the determination of moisture content in soil [28], in the food industry for the verification of the vegetable oil types [10], for non-destructive evaluation of an activated carbon [29], for monitoring of Pseudomonas Aeruginosa concentration [12], and in the healthcare industry [13], for example for real- time monitoring of 
glucose in diabetic patients [30,31] and for non-invasive monitoring of bodily fluids $[32,33]$.

When an aqueous sample with various type and concentration of residual antibiotics is placed into the vicinity of the electromagnetic field, it interacts with EM waves in a unique manner, which can be specifically correlated with the properties of this solution. The noninvasive and rapid nature demonstrated in other works encourages the application of the technique for the purpose of quantifying antimicrobials in aquaculture.

\section{Materials and Methods}

\section{Analyte Solutions Preparation}

Stock antibiotic solutions of enrofloxacin and norfloxacin were prepared by dissolving $10.0 \mathrm{mg}$ of each individual antibiotic in 1,000 $\mathrm{mL}$ of deionised water to achieve a final concentration of $10 \mathrm{mg} / \mathrm{L}(10$ $\mathrm{ppm}$ ). Each working antibiotics solutions were prepared by appropriate dilution of aliquots of the stock antibiotic solutions with deionised water.

\section{Microwave Cavity Design and Measurement Setup}

To assess the feasibility of using electromagnetic sensing approach for real-time residual antibiotics concentration monitoring, a comprehensive set of experiments was conducted. A bespoke twoport resonant cavity was used. Microwave cavities are widely used for characterising the properties of materials [29,31,34-36] and are usually made by shortening the two ends of a waveguide segment in which the electric field and magnetic field oscillate in phase perpendicular to each other and perpendicular to the direction of energy propagation. Electromagnetic waves transport the electrical power through the waveguide., Depending on the frequency, the dimensions and the material properties inside a waveguide, electric and magnetic field can take several different forms (electromagnetic (TEM) modes), such as transverse electric (TE) and transverse magnetic (TM).

A cavity will resonate when it is excited at an appropriate frequency; depending on the resonant mode and frequency, the distribution of the electric (and magnetic) fields will vary, effecting the interaction between the sample under test and the electromagnetic field(s) within the cavity. Figure 1 demonstrates the dominant electric field distribution at $10 \mathrm{GHz}$ for the cylindrical microwave cavity utilised in the work, as modelled using Ansys High Frequency Structure Simulation (HFSS) software.

Analysis using microwaves is non-ionising with a low power output of approximately $1 \mathrm{~mW}(0 \mathrm{dBm})$. They have a good penetration depth and associated equipment can be portable for real-time use at the pointof-control in aquaculture applications. The multi-parameter nature of wide band microwave analysis can provide unique signal spectrum signatures. Typically, these would be in the form of a reflected signal $S_{11}$ or/and a transmitted signal $\left|S_{21}\right|$, which are influenced by parameters such as conductivity and permittivity. Permittivity relates to a material's ability to transmit an electric field and is a complex value which varies with frequency, and accounts for the energy stored by a material $\left(\varepsilon^{\prime}\right)$ as well as any losses of energy ( $\left({ }^{\prime \prime}\right)$ which might occur.

The experimental setup used in this work with Rohde and Schwarz ZVA24 vector network analyser (VNA) connected to a microwave cavity with antibiotic-containing test water sample is illustrated in Figure 2. The microwave cavity was excited by a circular patch antenna placed inside it. The resonant modes occur when the electric and magnetic fields form standing waves, which depend on the internal dimensions

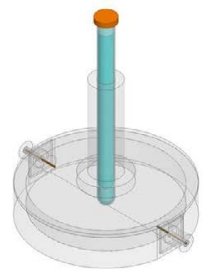

a)

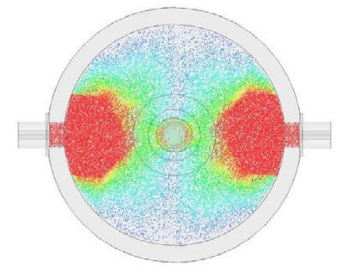

b)

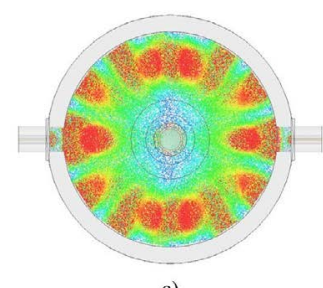

c)
Figure 1: a) 3D model of microwave cavity used in this work, produced using Ansys HFSS and electric field distributions at b) $1.5 \mathrm{GHz}$ and c) $10 \mathrm{GHz}$ from the top view. The model includes a representative control sample, with distilled water in a polypropylene test tube

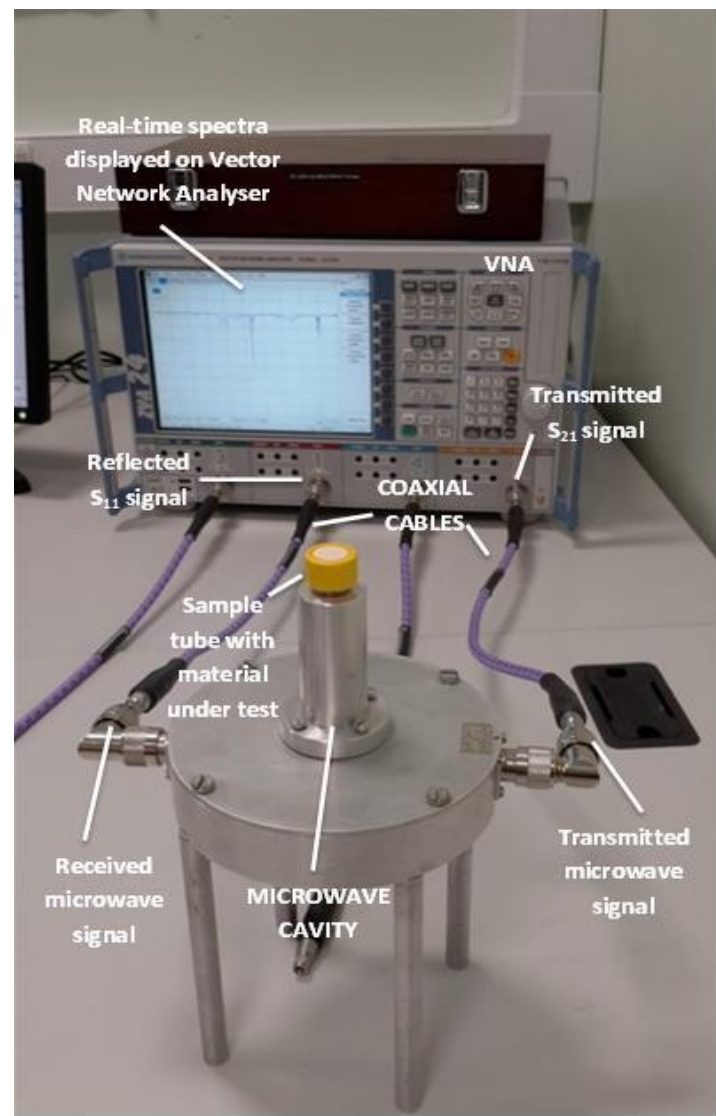

Figure 2: Experimental setup: Vector Network Analyser connected to a bespoke microwave cavity with antibiotic-containing test water sample measured in real time

of the cavity and the dielectric properties of the antibiotics present in water solution.

Spectral data was captured in the frequency range of $0.01-15 \mathrm{GHz}$ for the reflected $\left(\mathrm{S}_{11}\right)$ and transmitted $\left(\mathrm{S}_{11}\right)$ signals, with each measurement represented by 60,000 discrete data points. All the measurements were performed at a constant temperature of $18^{\circ} \mathrm{C}$, with all the samples being $15 \mathrm{ml}$ in volume for consistency. Each solution sample was measured at least 7 times to ensure repeatability and reproducibility or the results, and the average spectra and analysed, as detailed in the next section.

\section{Results and Discussion}

Measured sample had a unique response to the microwave signal resulting in resonant peaks occurring at different frequencies. Figure 3 illustrates the average spectra from each measured analyte solution. The discrepancy between each sample measurement was $<5 \%$, and could be due to imperfection of manual sample loading process, as it was shown 
that the system is sensitive to water volume changes [37]. This particular feature makes the developed microwave sensor an attractive option for real-time monitoring of residual antibiotics type and concentration in water.

Notably, the variation due to the change in signal caused by the varying antibiotic concentration is significantly greater than the measurement error. Once a change in microwave spectra due to antibiotic concentration is verified, one may narrow down the measurement frequency range and choose a limited number of points that would serve as a reference. Thus, plotting a calibration curve for $12 \mathrm{ml}$ solutions placed in the bespoke microwave cavity at a selected frequency of $10.02 \mathrm{GHz}$ resulted in a linear dependence of the $S_{21}$ transmitted microwave signal on ENR concentration, as is shown in Figure 4.

As one can see from Figure 4 and Figure 5, the microwave cavity exhibited linear decrease in the transmitted signal amplitude, $S_{21}$ at $10.02 \mathrm{GHz}\left(\mathrm{R}^{2}=0.9946\right)$ and reflected signal amplitude $\mathrm{S}_{11}$ at $1.52 \mathrm{GHz}$ $\left(\mathrm{R}^{2}=0.8821\right)$ respectively with increasing concentration of enrofloxacin. Measurements of norfloxacin (NOR) at the same frequencies produced different microwave spectra, as illustrated by a calibration curve at 1.52 $\mathrm{GHz}$ and no linear dependence was present. These results verify that the proposed approach of using microwave spectroscopy for real-time antimicrobial monitoring provides both reliability of the measurements and is selective, namely both the type and the concentration of antibiotics can be assessed.
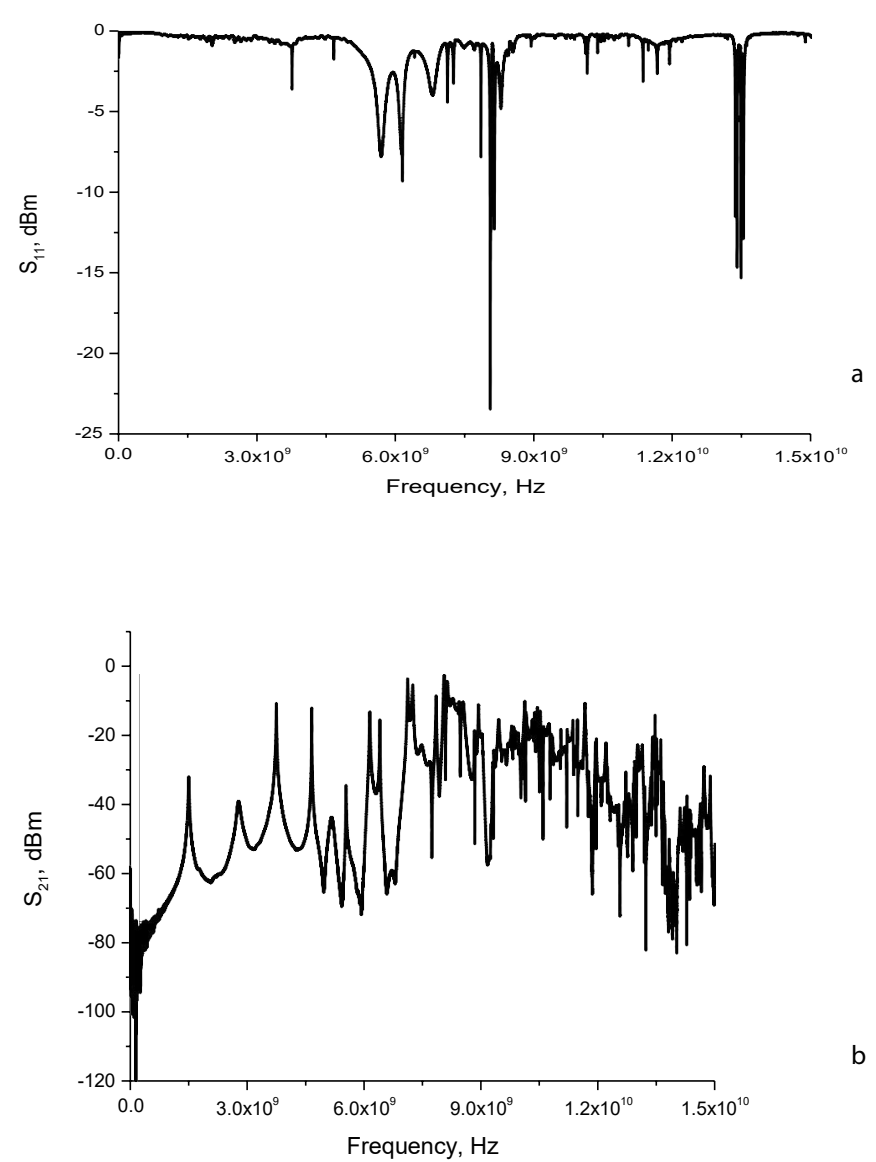

Figure 3: Typical microwave spectra for $50 \mathrm{ppb}$ concentration of enrofloxacin in $0.001-15 \mathrm{GHz}$ frequency range: a) reflected power $\mathrm{S}_{11}$ and $\mathrm{b}$ ) transmitted power $\mathrm{S}_{21}$

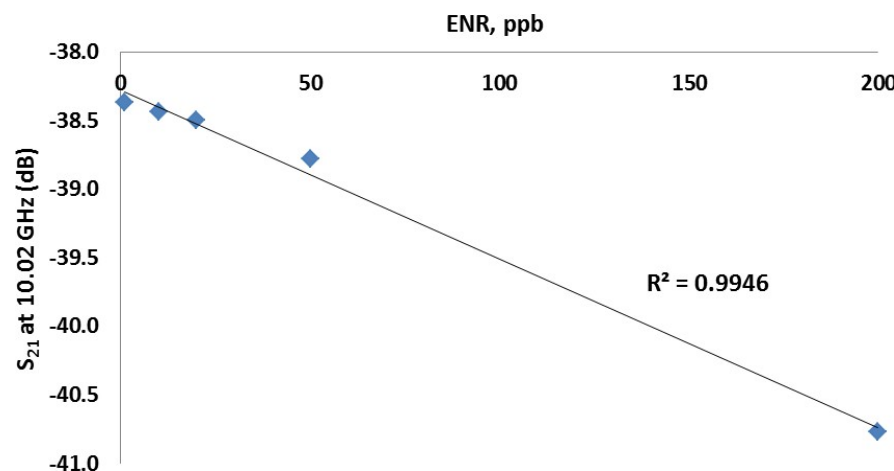

Figure 4: Dependence of the $S_{21}$ transmitted microwave signal on ENR concentration at $10.02 \mathrm{GHz}$ recorded for $12 \mathrm{ml}$ solutions placed in a bespoke microwave cavity

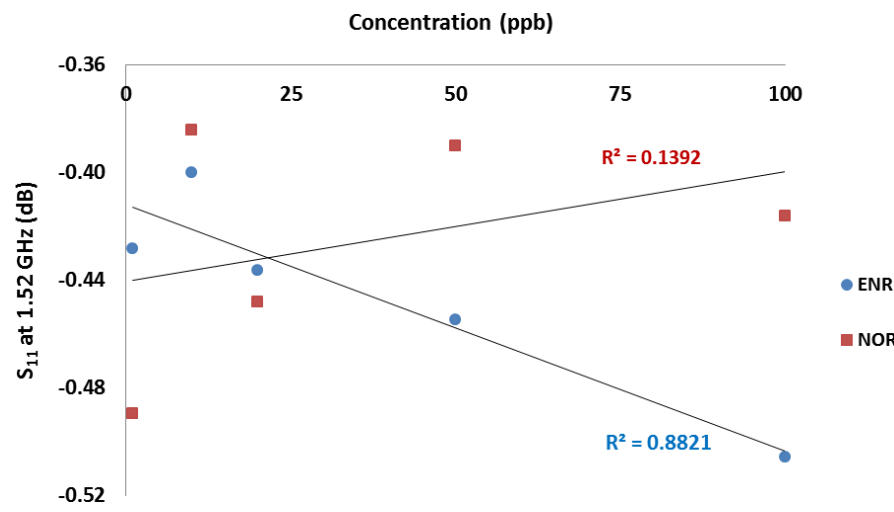

Figure 5: Dependence of the $S_{11}$ received microwave signal on ENR and NOR concentration at $1.52 \mathrm{GHz}$ recorded for $12 \mathrm{ml}$ solutions placed in a bespoke microwave cavity

Importantly, once the frequency range where the system is selectively sensitive to a particular antibiotic is known, the bulky and expensive VNA equipment could be replaced with custom-made hand-held electronics units for portable applications [13], where the measurements can take place anywhere and provide instant results.

Thus, substantial changes in the microwave spectra caused by the solutions with varying antibiotic concentrations were recorded and these can be used as an alternative to the traditional optical measurement method of an unknown analyte composition. This method is nondestructive, it provides real-time responses and the microwave power used is in the order of $1 \mathrm{~mW}$, which is non- ionising radiation, less than commonly used even in the mobile phones, and thus has negligible deleterious effect on the material being measured. Another advantage of this method is that there are no additional chemicals required to reveal the concentration of the analyte solution. The proposed system can potentially be integrated into an industrial production line or at any aquaculture industry check-point to automatically assess the composition of solutions in real time and manage the processes accordingly.

Notably, the sensors' response to air was used as the original benchmark positions. After each measurement of the analyte solution were performed and the sample was removed from the sensor surface, once the sensor was in contact only with air again, its response was identical to a benchmark measurements. This confirms that the developed microwave sensor is reliable, re-usable and thus a sustainable solution for precise in-situ method for industrial process monitoring, where real-time information on solutions composition is essential. 
The response of the sensor to other analyte solution types and concentrations is being explored and a database of these microwave signature spectra is being compiled, which can later be used for online process control in a broad range of industrial applications in the wastewater industry, chemical and pharmaceutical production lines. Further, the prototype system will be tested in real industrial settings and a challenge of differentiating between multiple water pollutants possibly present in a sample and determining their concentrations would emerge. The system can be scaled down for portable applications. The real-time nature of the measurements makes the suggested approach a valuable alternative to mainly lab-based method of health and safety and quality control in food industry, in aquaculture and environment.

\section{Conclusion}

The precise quantity of antimicrobials used in food production globally is difficult to estimate, but the evidence suggests that it is at least as great as the amount used by humans. This paper communicates the experimental results of using a bespoke microwave cavity sensor for real-time determination of antibiotic concentrations, in particular enrofloxacin and norfloxacin. A comprehensive set of complementary experiments using optical and microwave detection methods confirmed the potential of this novel sensing approach to serve as an alternative method of residual antibiotics concentration monitoring in a wide range of applications, including the food industry and environmental monitoring.

\section{References}

1. Smith P (2008) Antimicrobial resistance in aquaculture. Rev Sci Tech 27: 243-264. [Crossref]

2. Marshall BM, Levy SB (2011) Food animals and antimicrobials: Impacts on human health. Clin Microbiol Rev 24: 718-733. [Crossref]

3. Burridge L, Weis JS, Cabello F, Pizarro J, Bostick K (2010) Chemical use in salmon aquaculture: A review of current practices and possible environmental effects. Aquaculture 306: 7-23.

4. Lupo A, Coyne S, Berendonk TU (2012) Origin and evolution of antibiotic resistance: The common mechanisms of emergence and spread in water bodies. Front Microbiol 3: 18 . [Crossref]

5. Naviner M, Gordon L, Giraud,E, Denis M, Mangion C, et al. (2011) Antimicrobial resistance of aeromonas spp. Isolated from the growth pond to the commercial product in a rainbow trout farm following a flumequine treatment. Aquaculture 315: 236-241.

6. Wegener HC (2003) Antibiotics in animal feed and their role in resistance development. Curr Opin Microbiol 6: 439-445. [Crossref]

7. Dorival-García N, Zafra-Gómez A, Cantarero S, Navalón A, Vílchez JL (2013) Simultaneous determination of 13 quinolone antibiotic derivatives in wastewater samples using solid-phase extraction and ultra performance liquid chromatographytandem mass spectrometry. Microchem J 106: 323-333.

8. Korostynska O, Mason A, Al-Shamma'a AI (2013) Flexible microwave sensors for real-time analysis of water contaminants. J Electromagnetic Waves Appl 27: 20752089.

9. Korostynska O, Mason A, Ortoneda-Pedrola M,Al-Shamma'a A(2014) Electromagnetic wave sensing of no3 and cod concentrations for real-time environmental and industrial monitoring. Sens Actuators B Chem 198: 49-54.

10. Korostynska O, Blakey R, Mason A, Al-Shamma'a A (2013) Novel method for vegetable oil type verification based on real-time microwave sensing. Sens Actuators A Phys 202: 211-216.

11. Mason A, Abdullah B, Muradov M, Korostynska O, Al-Shamma'a A, et al. (2016) Theoretical basis and application for measuring pork loin drip loss using microwave spectroscopy. Sensors 16: 182. [Crossref]

12. Blakey R, Nakouti I, Korostynska O, Mason A, Al-Shamma'a A (2013) Real-time monitoring of pseudomonas aeruginosa concentration using a novel electromagnetic sensors microfluidic cell structure. IEEE Trans Biomed Eng 60: 3291-3297.
13. Mason A, Korostynska O, Ortoneda-Pedrola M, Shaw A, Al-Shamma'a A (2013) A resonant co-planar sensor at microwave frequencies for biomedical applications. Sens Actuators A Phys 202: 170-175.

14. Poirel L, Rodriguez-Martinez JM, Mammeri H, Liard A, Nordmann P (2005) Origin of plasmid-mediated quinolone resistance determinant qnra. Antimicrob Agents Chemother 49: 3523-3525.

15. Arber W (2014) Horizontal gene transfer among bacteria and its role in biological evolution. Life (Basel) 4: 217-224. [Crossref]

16. Taylor NG, Verner-Jeffreys DW, Baker-Austin C (2011) Aquatic systems: Maintaining, mixing and mobilising antimicrobial resistance? Trends Ecol Evol 26: 278-284.

17. Le TX, Munekage Y, Kato SI (2005) Antibiotic resistance in bacteria from shrimp farming in mangrove areas. Science of The Total Environment 349: 95-105. [Crossref]

18. De Alwis HG, Girard L, Kijak PJ, Rummel N (2013) Determination of erythromycin in medicated salmonid fish feed by liquid chromatography and uv spectroscopy. Int Journal of AOAC 96: 1233-1238. [Crossref]

19. Chafer-Pericas C, Maquieira A, Puchades R, Miralles J, Moreno A (2010) In Fast screening immunoassay of sulfonamides in commercial fish samples, Tiergartenstrasse 17, Heidelberg, D-69121, Germany; Springer Verlag: Tiergartenstrasse 17, Heidelberg, D-69121, Germany, pp: 911-921.

20. Almeida SAA, Amorim LR, Heitor AH, Montenegro MCBSM, Barbosa J, et al. (2011) Rapid automated method for on-site determination of sulfadiazine in fish farming: A stainless steel veterinary syringe coated with a selective membrane of pvc serving as a potentiometric detector in a flow- injection-analysis system. Anal Bioanal Chem 401: 3355-3365.

21. Kim B, Lim D, Jin HJ, Lee HY, Namgung S, et al. (2012) Family-selective detection of antibiotics using antibody-functionalized carbon nanotube sensors. Sens Actuators B Chem 166-167: 193-199.

22. Carrasco S, Benito-Pena E, Walt DR, Moreno-Bondi MC (2015) Fiber-optic array using molecularly imprinted microspheres for antibiotic analysis. Chemical Science 6: 3139-3147. [Crossref]

23. Kapilevich B, Litvak B (2007) In Microwave sensor for accurate measurements of water solution concentrations, APMC Asia-Pacific Microwave Conference, Bangkok, pp: $1-4$.

24. Boon JD, Brubaker JM (2008) In Acoustic-microwave water level sensor comparisons in an estuarine environment, OCEANS, Quebec, Canada, pp: 1-5.

25. Jackson B, Jayanthy T (2010) In A novel method for water impurity concentration using microstrip resonator sensor, Recent Advances in Space Technology Services and Climate Change (RSTSCC), Chennai, India, pp: 376-379.

26. Bernou C, Rebière D, Pistré J (2000) Microwave sensors: A new sensing principle Application to humidity detection. Sens Actuators B Chem 68: 88-93.

27. Nacke T, Barthel A, Pflieger C, Pliquett U, Beckmann D, et al. (2010) In Continuous process monitoring for biogas plants using microwave sensors, 12th Biennial Baltic Electronics Conference (BEC) Tallinn, Estonia, pp: 239-242.

28. You KY, Salleh J, Abbas Z, You LL (2010) In A rectangular patch antenna technique for the determination of moisture content in soil, Progress in Electromagnetics Research Symposium, PIERS 2010 Cambridge, Electromagnetics Academy: Cambridge, MA USA, pp: 850-854.

29. Mason A, Korostynska O, Wylie S, Al-Shamma'a AI (2014) Non-destructive evaluation of an activated carbon using microwaves to determine residual life. Carbon 67: 1-9.

30. Korostynska O, Arshak A, Creedon P, Arshak K, Wendling L, et al. (2009) In Glucose monitoring using electromagnetic waves and microsensor with interdigitated electrodes, IEEE Sensors Applications Symposium, SAS, New Orleans, LA, USA, pp: 34-37.

31. Mason A, Wylie S, Thomas A, Keele H, Shaw A, et al. (2010) Hepa filter material load detection using a microwave cavity sensor. International Journal on Smart Sensing and Intelligent Systems 3: 322-337.

32. Al-Shamma'a A, Mason A, Shaw A (2012) Patent: Non-invasive monitoring device. US2012150000 (A1), WO2010131029 (A1), EP2429397 (A1).

33. Mason A, Shaw A, Al-Shamma'a A (2012) A co-planar microwave sensor for biomedical applications. Procedia Eng 47: 438-441.

34. Al-Kizwini MA, Wylie SR, Al-Khafaji DA, Al-Shamma'a AI (2013) The monitoring of the two phase flow-annular flow type regime using microwave sensor technique. Measurement (Lond) 46: 45-51.

35. Al-Shamma'a AI, Pandithas I, Lucas J (2001) Low-pressure microwave plasma ultraviolet lamp for water purification and ozone applications. J Phys D Appl Phys 34: $2775-2781$ 
Korostynska O (2018) Tackling water pollution: real-time monitoring of residual antimicrobials concentration in aquaculture with microwave spectroscopy

36. Blakey RT, Mason A, Al-Shamma'a A, Rolph CE, Bond G (2013) Dielectric characterisation of lipid droplet suspensions using the small perturbation technique. In Advancement in sensing technology, Mukhopadhyay SC, Jayasundera KP, Fuchs A, Eds. Springer Berlin, 1: 81-91
37. Jader, M.A, Korostynska, O, Mason, A, A1-Shamma'A, A.I. In Non-destructive volume and thickness measurements with planar microwave sensors, 2013 IEEE 33rd International Scientific Conference Electronics and Nanotechnology, ELNANO 2013, April 16, 2013 - April 19, 2013, Kyiv, Ukraine, 2013; IEEE Computer Society: Kyiv, Ukraine, pp: 465-468.

Copyright: $@ 2018$ Korostynska O. This is an open-access article distributed under the terms of the Creative Commons Attribution License, which permits unrestricted use, distribution, and reproduction in any medium, provided the original author and source are credited. 